\title{
KLEIN PARADOX FOR BOUND STATES - A PUZZLING PHENOMENON
}

\author{
Nagalakshmi A. Rao* \& B.A. Kagali**
}

\begin{abstract}
While Klein paradox is offen encountered in the context of scattering of relativistic particles at a potential barrier, we presently discuss a puzzling situation that arises with the Klein-Gordon equation for bound states. With the usual minimal coupling procedure of introducing the interaction potential, a paradoxical sifuation results when the "hill" becomes a "well", simulating a bound state like situation. This phenomenal phenomenon for bound states is contrary to the conventionol wisdom of quantum mechanics and is analogous to the well-known. Klein paradox, a generic property of relativistic wove equations.
\end{abstract}

\section{Introduction}

In non-relativistic quantum mechanics, the scattering of an electron by a potential barrier is known to be one of the simplest solvable problems. However, a similar problem with a potential step or a barrier in relativistic quantum mechanics, often results in paradoxical situations, called the Klein Paradox.

* Department of Physics, Government Science College, Bangalore -560001, Kamataka, India na rao@hotmail.com

** Department of Physics, Bangalore University, Bangalore-560056, Karnataka, India bakagali@hotmail.com 
In the original work of Klein, [I] [1929], electrons incident on a large potential step was addressed. The problem was treated with the Dirac equation and it was found that for large potentials, $V(x)>E+m c^{2}$, the reflection coefficient, $R_{S^{\prime}}$ exceeds unity while the transmission coefficient, $T_{s}$, becomes negative. This suggested that more particles are reflected by the step than are incident on it. Such a puzzling situation contradicting non-relativistic expectation was termed Klein Paradox. This phenomenon suggests the possibility of creation of particle and antiparticle pairs by a Klein step.

Several authors ${ }^{[2-6]}$ have discussed Klein Paradox under various circumstances. Considering a potential step with sharp boundaries, Biorken ${ }^{[7]}$ has illustrated that a weak potential having decaying exponential solutions inside the potential region leads to undamped oscillatory solutions for potentials exceeding $\left(E+m c^{2}\right)$, consistent with the original version of Klein Paradox. However, Greiner, ${ }^{[8]}$ on the basis of the group velocity treatment has illustrated an unexpected largeness of the transmission coefficient. While Brojken's explanation of Klein Paradox is essentially based on pair production, Greiner's representation is that of single-particle interpretation.

Similar results exist for Klein Gordon particles as well. Guang Joing et al. ${ }^{[9]}$ have shown that the Klein-Gordon equation with a step potential in minimal coupling exhibits the Klein Paradox at the one-particle level. Rubin Landau ${ }^{100}$ has given a reasonable explanation of the Klein Paradox based on particle-antiparticle pair production.

In the following section, we discuss the appearance of a paradoxical result in the context of bound states.

\section{The Klein-Gordon Equation with a Potential}

The time-independent one-dimensional Klein-Gordon equation for a general potential introduced as a vector field, may well be written as

$$
\left\{\frac{d^{2}}{d x^{2}}+\frac{(E-V(x))^{2}-m^{2} c^{4}}{c^{2} \hbar^{2}}\right\} \psi(x)=0
$$

This equation may be cast into the form of Schrodinger equation as

$$
\frac{d^{2}}{d x^{2}}+\frac{2 m}{\hbar^{2}}\left(E_{\text {eff }}-V_{\text {eff }}\right) \psi(x)=0,
$$


with

$$
E_{\mathrm{eff}}=\frac{E^{2}-m^{2} c^{4}}{2 m c^{2}}
$$

and

$$
V_{\text {eff }}=\frac{2 E V(x)-V^{2}(x)}{2 m c^{2}}
$$

The concept of effective energy and effective potential used to simulate the properties of relativistic wave equations leads to paradoxical results.

We first review the finite square well problem and then address the case of potential hill.

\section{A. Potential Well}

For an attractive square well potential defined by

$$
V(x)=\left\{\begin{array}{l}
-V_{0} \text { for }|x| \leq 0 \\
0 \text { for }|x|>0
\end{array}\right.
$$

the effective potential takes the form

$$
V_{\text {eff }}(x)=\frac{-2 E V_{0}-V_{0}^{2}}{2 m c^{2}} \text { for }|x| \leq a
$$

and vanishes elsewhere. Considering positive energy states, the effective potential looks like another square well potential and therefore, so long as $E_{\text {eff }}<0$ or $E<m c^{2}$, bound states are possible. This result is quite reasonable.

\section{B. Potential Hill}

We now consider a potential barrier defined by

$$
V(x)=\left\{\begin{array}{l}
-V_{0} \text { for }|x| \leq a \\
0 \text { for }|x|>a
\end{array}\right.
$$

which is not the usual Klein step, but has befter-defined boundaries. The effective potential for a 'hill' takes the form 


$$
V_{\mathrm{eff}}(x)=\frac{-2 E V_{0}-V_{0}^{2}}{2 m c^{2}}
$$

the effective energy remains the same. It is trivial to check that

$$
\left(E_{\mathrm{eff}}-V_{\text {eff }}\right)=\left\{\begin{array}{cc}
\frac{E^{2}-m^{2} c^{4}+V_{0}^{2}-2 E V_{0}}{2 m c^{2}} & \text { for }|x| \leq a \\
\frac{E^{2}-m^{2} c^{4}}{2 m c^{2}} & \text { for }|x|>a
\end{array}\right.
$$

Interestingly, $V_{\text {eff }}$ can be positive, zero or even negative for a range of values of the barrier height $V_{0}$.

For, $V_{0}<2 m c^{2}, V_{\text {eff }}$ remains positive, and the problem is analogous to a typical scattering problem.

For $V_{0}=2 m c^{2}$, the effective potential vanishes and the barrier becomes supercritical.

However a puzzling situation arises for a barrier height exceeding $2 m c^{2}$. As is seen from Eqn. (8), the effective potential becomes negative and thus a 'hill' is transformed into a 'well'. For $\left(V_{0}>2 m c^{2}\right)$, particles, instead of being scattered by the potential "hill" are trapped inside the simulated "well". This means that bound states are possible for strong barriers. Such a paradoxical situation may be called the Klein Paradox for bound states.

More importantly, it may be inferred that the potential hill need not be only of the square type for such anomalous bound states. The actual number of the bound states and the energies, however, will depend on the shape and size of the hill. These trivial results may be worked out from the standard procedures of quantum mechanics.

\section{Results and Discussion}

While the original version of Klein Paradox concerns scattering situation of Dirac particles at a potential step, we have shown that an analogous paradoxical situation arises even for bound states. With the usual minimal coupling procedure of introducing interaction, the Klein-Gordon equation leads to bound states for finitely extended potential hills, contrary to the conventional wisdom of quantum mechanics. So long as the effective vector potential remains positive, $\left(V<2 m c^{2}\right)$ for repulsive potentials, the situation is similar to a typical scattering problem. As the potential 
becomes stronger and stronger, exceeding the limiting value $E+m c^{2}$ the 'hill' becomes a 'well' simulating a bound state-like situation. Surprisingly, a typical scattering problem is transformed into a bound state problem. Thus Klein Paradox is retrieved for bound states in the case of a strong repulsive, finite ranged barrier.

Interestingly, a paradoxical situation like this does not arise for pure scalar repulsive potentials. In such a case, a barrier is transformed into another barrier, no matter how strong or weak the potential is. The paradoxical result for bound state illustrates that Klein-Gordon equation is reasonable even at the one particle level.

\section{Acknowledgements}

One of the authors (N.A. Rao) is grateful to University Grants Commission and the Department of Collegiate Education in Karnataka for the award of Teacher Fellowship under the Faculty Improvement Programme. Thanks are extended to the Director of Collegiate Education in Karnataka, Prof. K.V. Kodandaramaiah for his encouragement.

\section{References}

1. Klien O.Z. Physics, 53 (1929) 157-165.

2. Mark J. Thomson and Bruce H.J. McKellar, The solution of the Dirac equation for high squre barrier, Am. J. Phys. 59(4) (1991) 340-346.

3. Francisco Dominguez-Adame, A relativistic interaction without Klein Paradox, Phys. Left. A 162 (1992) 18-20.

4. Holstein B.R., Klein Poradox, Am. J. Phys. 69 (1999) 507-512. 5. Calogeracos A and N. Dombey, Klein tunneling and Klein Paradox, Int J.M. Phys. A 14 (4)
(1999) 631-643. 6. Antonio S de Castro, $(n+1)$ dimensional Dirac equation and the Klein Paradox, Am.J. Phys.
$69(10)(2001), 1111-1112$.

7. James D. Bjorken and Sidney D. Drell, Relativistic Quantum Mechanics, Mc Graw Hill Book Company, NY, (1964) pp 40.43.

8. Greiner, Walter, Relativistic Quantum Mechanics-Wave Equations, Springer-Verlag, (1995).

9. Gaung-Jiong Ni, Weimin Zhou and Jun Yan, Klein Paradox and antiparticle.

10. Rubin H. Landau, Quantum Mechanics II A Second Course in Quantum Theory, A Wiley Interscience Publication, New York, 1996. 